\title{
New data on vitamin D deficiency and mortality
}

\author{
Ginter $E^{1}$, Simko $V^{2}$ \\ Slovak Medical University, Bratislava, Slovakia. ginter.emil@mail.t-com.sk
}

PubMed registered in 2014 about 26,000 papers on vitamin D (D) published in the past ten years. In the review in 2009 (1) we pointed out that the non-skeletal, metabolic effects of $D$ have been subject of intensive research only in the last two decades. $\mathrm{D}$ insufficiency and deficiency are clearly associated with suboptimal health. Past two years witnessed several papers suggesting a shortening of the average life expectancy (LE) in populations exposed to $\mathrm{D}$ deficiency. Let us review some of the most relevant reports.

The blood level of 25-hydroxy-vitamin D (25(OH)D) is a reliable indicator of body stores of this important biological compound. In the extensive study (2) researchers compared levels of D in 9,578 individuals age 50 to 74 with their mortality over 9.5 years. The relative risk of overall mortality of subjects with vitamin D deficiency [25(OH)D concentrations $<30 \mathrm{nmol} / \mathrm{L}$ ] or vitamin D insufficiency [25(OH)D] concentrations from 30 to $50 \mathrm{nmol} / \mathrm{L}$ ) was significantly increased to 1.71 and 1.17 , respectively in these low D level subjects, as opposed to individuals with sufficient $25(\mathrm{OH}) \mathrm{D}$ concentrations ( $>50 \mathrm{nmol} / \mathrm{L}$ ). Vitamin D deficiency was also associated with an increased cardiovascular mortality (1.39) and cancer mortality (1.42).

Remarkable are conclusions of a very large international group of researchers published in 2014 (3). These were based on analysis of specific relative risks of mortality from 73 cohort studies (849,412 participants) and 22 randomized controlled trials (30,716 participants). In the primary prevention observational studies, comparing bottom versus top thirds of baseline circulating 25(OH)D distribution, pooled relative risks were 1.35 for death from cardiovascular disease, 1.14 for death from cancer, and 1.35 for all cause mortality. The evidence from observational studies indicates inverse associations of circulating 25(OH)D with risks of death due to cardiovascular disease, cancer, and other causes.

Another very recent information from 2014 (4) is the metaanalysis of individual participant data of eight prospective cohort studies from Europe and the USA. This included 26018 men and women aged 50-79 years. For each cohort and analysis, 25(OH) D quintiles were defined. Figure 1 shows a comparison of 25-hydroxyvitamin D quintiles with respect to all-cause mortality outcomes. It is obvious that lower levels of vitamin D were associated with a higher mortality. Risk ratios for cardiovascular mortality were similar in magnitude to that for all-cause mortality. Analyses using all quintiles suggest curvilinear, inverse, dose-response

${ }^{1}$ Emeritus, Slovak Medical University, Bratislava, Slovakia, ${ }^{2}$ State University New York, Downstate Medical Centre at Brooklyn, USA

Address for correspondence: E. Ginter, RND, DSc, Racianska 17, SK83102 Bratislava, Slovakia.

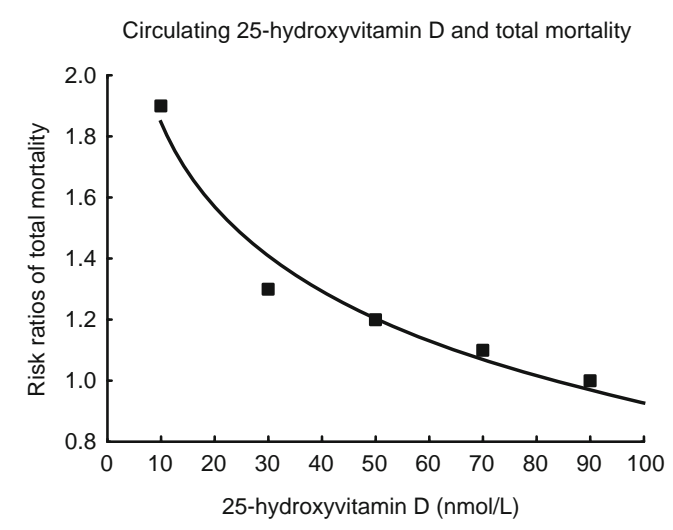

Fig. 1. Dose-response trend of pooled effect estimates for the comparison of 25-hydroxyvitamin D quintiles (top quintile as reference) with respect to total mortality outcomes. According to Schöttker et al (4).

curves for the aforementioned relationships. No strong age, sex, seasonal, or country specific differences were detected.

\section{Conclusions}

Despite levels of 25(OH)D strongly vary with country, sex and season, the associations between deficient 25(OH)D levels and a significant increase of all-cause mortality were remarkably consistent. The results from a long term trials addressing longevity are being awaited before vitamin $\mathrm{D}$ supplementation can be recommended in most individuals with low 25(OH)D levels. Any discussion on vitamin D supplementation has to start with the essential role of ultraviolet light on biosynthesis of D in the skin.

\section{References}

1. Ginter E, Simko V. Vitamin D deficiency, atherosclerosis and cancer. Bratisl Lek Listy 2009; 110 (12): 751-756.

2. Schöttker B, Haug U, Schomburg L et al. Strong associations of 25-hydroxyvitamin D concentrations with all-cause, cardiovascular, cancer, and respiratory disease mortality in a large cohort study. Am J Clin Nutr 2013; 97: 782-793.

3. Chowdhury R, Kunutsor S, Vitezova A et al. Vitamin D and risk of cause specific death: systematic review and meta-analysis of observational cohort and randomised intervention studies. BMJ 2014; 348: g1903.

4. Schöttker B, Jorde R, Peasey A et al. Vitamin D and mortality: metaanalysis of individual participant data from a large consortium of cohort studies from Europe and the United States. BMJ 2014; 348: g3656.

Received July 1, 2014. Accepted January 23, 2015. 